(1)

CrossMark

\title{
Upfront combination therapy reduces right ventricular volumes in pulmonary arterial hypertension
}

\author{
Mariëlle C. van de Veerdonk ${ }^{1,2}$, Anna E. Huis in t Veld ${ }^{1}$, J. Tim Marcus ${ }^{3}$, \\ Nico Westerhof ${ }^{1}$, Martijn W. Heymans ${ }^{4}$, Harm-Jan Bogaard ${ }^{1}$ and \\ Anton Vonk-Noordegraaf ${ }^{1}$
}

Affiliations: 'Department of Pulmonary Diseases, Institute for Cardiovascular Research (ICaR-VU), VU University Medical Center, Amsterdam, The Netherlands. ${ }^{2}$ Department of Cardiology, Institute for Cardiovascular Research (ICaR-VU), VU University Medical Center, Amsterdam, The Netherlands. ${ }^{3}$ Department of Physics and Medical Technologies, Institute for Cardiovascular Research (ICaR-VU), VU University Medical Center, Amsterdam, The Netherlands. ${ }^{4}$ Department of Epidemiology and Biostatistics, Institute for Cardiovascular Research (ICaR-VU), VU University Medical Center, Amsterdam, The Netherlands.

Correspondence: A. Vonk Noordegraaf, VU University Medical Center, Department of Pulmonary Diseases, De Boelelaan 1117, 1081 HV Amsterdam, The Netherlands. E-mail: a.vonk@vumc.nl

@ERSpublications

Upfront combination therapy improves right ventricular volumes in pulmonary arterial hypertension patients http://ow.ly/WPqY30bS2Ee

Cite this article as: van de Veerdonk MC, Huis in t Veld AE, Marcus JT, et al. Upfront combination therapy reduces right ventricular volumes in pulmonary arterial hypertension. Eur Respir J 2017; 49: 1700007 [https://doi.org/10.1183/13993003.00007-2017].

ABSTRACT In pulmonary arterial hypertension (PAH), upfront combination therapy is associated with better clinical outcomes and a greater reduction in N-terminal pro-brain natriuretic peptide (NT-proBNP) than monotherapy. NT-proBNP levels reflect right ventricular (RV) wall stress, which increases when the right ventricle dilates. This study explored the impact of upfront combination therapy on RV volumes compared with monotherapy in $\mathrm{PAH}$ patients.

This retrospective study involved 80 incident PAH patients (New York Heart Association class II and III) who were treated with upfront combination therapy $(\mathrm{n}=35)$ (i.e. endothelin receptor antagonists (ERAs) plus phosphodiesterase-5-inhibitors (PDE5Is)) or monotherapy $(\mathrm{n}=45)$ (i.e. either ERAs or PDE5Is). All patients underwent right-sided heart catheterisation and cardiac magnetic resonance imaging at baseline and after 1year follow-up.

Combination therapy resulted in more significant reductions in pulmonary vascular resistance and pulmonary pressures than monotherapy. NT-proBNP was decreased by $\sim 77 \%$ in the combination therapy group compared with a $\sim 51 \%$ reduction after monotherapy $(\mathrm{p}<0.001)$. RV volumes and calculated RV wall stress improved after combination therapy (both $\mathrm{p}<0.001$ ) but remained unchanged after monotherapy (both $\mathrm{p}=\mathrm{NS}$ ). RV ejection fraction improved more in the combination therapy group than in the monotherapy group $(\mathrm{p}<0.001)$.

In PAH patients, upfront combination therapy was associated with improved RV volumes.

This article has supplementary material available from erj.ersjournals.com

Earn CME accreditation by answering questions about this article. You will find these at erj.ersjournals.com/journal/cme Received: Jan 032017 | Accepted after revision: March 112017

Support statement: This work was supported by the Netherlands CardioVascular Research Initiative: the Dutch Heart Foundation, Dutch Federation of University Medical Centres, the Netherlands Organisation for Health Research and Development, and the Royal Netherlands Academy of Sciences (CVON Phaedra). A. Vonk Noordegraaf was supported by a Netherlands Organisation for Scientific Research Vici grant (NWO-VICI 2002406). Funding information for this article has been deposited with the Crossref Funder Registry.

Conflict of interest: Disclosures can be found alongside this article at erj.ersjournals.com

Copyright OERS 2017 


\section{Introduction}

Pulmonary arterial hypertension (PAH) is characterised by abnormal pulmonary vascular remodelling resulting in chronic pressure overload of the right ventricle and ultimately the development of right ventricular (RV) failure and death $[1,2]$. The general treatment goal in patients with PAH is to reduce the load on the right ventricle in order to accomplish favourable RV adaptation, stable RV function and low mortality rates $[3,4]$. Treatment of PAH patients within New York Heart Association (NYHA) functional class II or III comprises either (1) initial single-agent therapy with endothelin receptor antagonists (ERAs) or phosphodiesterase-5-inhibitors (PDE5Is), or (2) the application of both agents (i.e. upfront combination therapy) [3]. Recently, it was shown in the Ambrisentan and Tadalafil in Patients with Pulmonary Arterial Hypertension (AMBITION) trial that upfront oral combination therapy resulted in a longer time to clinical failure and a greater reduction in N-terminal pro-brain natriuretic peptide (NT-proBNP) compared with upfront monotherapy [5]. Since changes in NT-proBNP reflect changes in RV wall stress [6, 7], these findings may be explained by either a reduction in pulmonary pressures or by more favourable RV remodelling. Indeed, it was recently observed that pulmonary pressures dropped significantly after upfront combination therapy [8]. However, to date, changes in RV volumes and wall thickness after combination therapy have not yet been explored. This could be of importance because RV dilatation is among the strongest predictors of mortality in PAH patients [9] and is an important determinant of RV wall stress [10]. Previous studies have demonstrated that although monotherapy leads to a decrease in pulmonary vascular resistance (PVR), it does not affect RV dilatation, and consequently RV wall stress remains high [11-15]. Based on the observation that NT-proBNP decreases more in the combination treatment group [5], we hypothesised that upfront combination therapy not only results in a greater decrease in PVR but will also lead to improvements in RV volumes, thereby reducing RV wall stress.

Therefore, the aim of the present study was to assess the therapeutic effects of upfront oral combination therapy on RV volumes in NYHA class II or III patients with idiopathic PAH (IPAH), heritable PAH (HPAH) or drug- and toxin-induced PAH (DPAH). PAH patients treated with upfront oral monotherapy were used as a control group.

\section{Methods}

Study design and patient selection

This study retrospectively analysed data from an ongoing prospective registry of newly diagnosed PAH patients admitted to the VU University Medical Center, Amsterdam, who routinely underwent right-sided heart catheterisation (RHC), cardiac magnetic resonance imaging (CMR), six-minute walk testing and blood sampling. Because the Medical Ethics Review Committee of the VU University Medical Center did not consider the study to fall within the scope of the Medical Research Involving Human Subjects (WMO) (approval number 2012288), an informed consent statement was not obtained.

Inclusion criteria for the present study were: (1) newly diagnosed patients with IPAH, HPAH or DPAH, (2) age $\geqslant 18$ years, (3) NYHA functional class II or III, (4) the use of oral PAH-specific medication consisting of ERA or PDE5I applied as either upfront monotherapy or dual combination therapy (i.e. initiated directly after diagnosis), (5) RHC and CMR performed at baseline and after 1 year of follow-up. Patients with a positive acute vasodilator challenge and/or patients treated with calcium channel blockers [3] were excluded from the analysis. Patients meeting the inclusion criteria were enrolled between August 2002 and July 2015, and totalled 114 patients. RHC and CMR were performed within a median time interval of 2 days. Nine patients died during the first year of follow-up and were excluded (two of these patients had received upfront combination therapy and seven had been treated with monotherapy). Nine of the 105 patients were excluded because of treatment with calcium channel blockers. In addition, 16 patients had no or insufficient CMR assessment at 1 year follow-up and could therefore not be included. In total, 80 patients fulfilled the study criteria and were included in the present study (figure 1). Thirty-five patients treated with upfront combination therapy were compared with 45 patients who received upfront monotherapy.

\section{Treatment regimens}

Application of PAH-targeted medical therapies was performed in line with the guidelines and according to the availability in the Netherlands. Since August 2002 and 2005, ERA (ambrisentan, bosentan, macitentan or sitaxentan) and PDE5I (sildenafil or tadalafil), respectively, have been available in the Netherlands. Patients were treated with upfront oral monotherapy (ERA or PDE5I) or upfront dual combination therapy (ERA plus PDE5I). Upfront monotherapy was defined as the application of one type of drug, initiated directly after diagnosis. Upfront combination therapy implies the application of two types of drugs (ERA plus PDE5I), both started at the exact same time point after diagnosis and up-titrated in the following 4-8 weeks. The treating physician decided which specific type of ERA or PDE5I was to be applied and whether a patient should receive upfront monotherapy or combination therapy. 


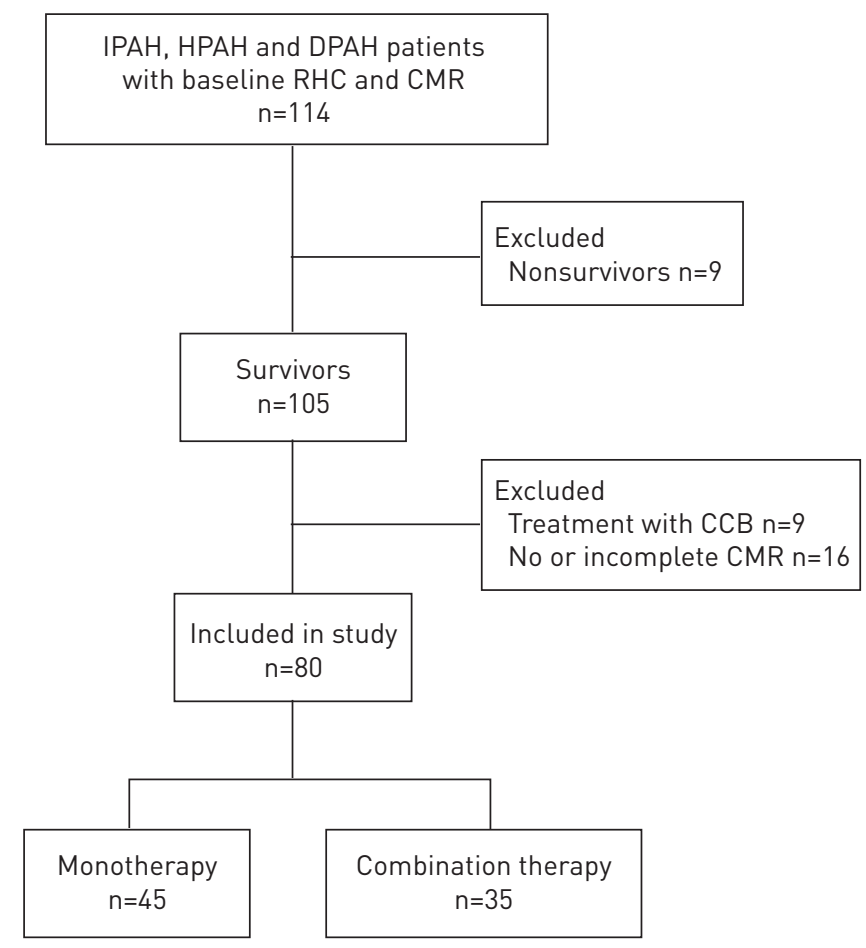

FIGURE 1 Study profile. CCB: calcium channel blockers; CMR: cardiac magnetic resonance imaging; DPAH: drug- and toxin-induced pulmonary arterial hypertension; HPAH: heritable pulmonary arterial hypertension; IPAH: idiopathic pulmonary arterial hypertension; RHC: right-sided heart catheterisation.

Dosing regimens were as follows: bosentan $62.5 \mathrm{mg}$ twice daily, increasing to $125 \mathrm{mg}$ twice daily after 4 weeks; ambrisentan $5 \mathrm{mg}$ once daily, increasing up to $10 \mathrm{mg}$ once daily if necessary; macitentan $10 \mathrm{mg}$ once daily without further up-titration; sitaxentan $100 \mathrm{mg}$ daily; sildenafil $20 \mathrm{mg}$ three times daily; tadalafil $20 \mathrm{mg}$ once daily, up-titrated up to $40 \mathrm{mg}$ once daily after 1 week.

All patients received anticoagulants, diuretics and oxygen therapy if needed. During follow-up, some patients went through one or multiple treatment regimens.

\section{Assessments}

Right-sided heart catheterisation

Haemodynamic assessment was performed with a 7F balloon-tipped flow-directed Swan-Ganz catheter (131HF7, Baxter Healthcare Corp, Irvine, CA, USA), inserted via the jugular or femoral vein during continuous electrocardiographic monitoring. The following parameters were measured: mean pulmonary arterial pressure (mPAP), right atrial pressure, pulmonary arterial wedge pressure (PAWP), heart rate and mixed venous oxygen saturation. Cardiac output (CO) was measured using the Fick method or thermodilution method. PVR was calculated as $80 \times(\mathrm{mPAP}-\mathrm{PAWP}) / \mathrm{CO}$. CO was indexed to body surface area (BSA), shown as cardiac index (CI).

\section{Cardiac magnetic resonance imaging}

CMR was performed on a Siemens 1.5-Tesla Sonata or 1.5-Tesla Avanto scanner (Siemens Medical Solutions, Erlangen, Germany), equipped with a 6-element phased-array receiver coil. Electrocardiographic-gated cine magnetic resonance (MR) imaging was performed using a balanced steady-state precession pulse sequence during repeated inspiratory breath-holds. CMR data acquisition was acquired according to our standard protocol [16]. After recording several localiser images, a variety of short-axis images covering the ventricles from base to apex were obtained with a typical slice thickness of $5 \mathrm{~mm}$ and an interslice gap of $5 \mathrm{~mm}$.

The short-axis images were post-processed by a blinded observer who analysed the ventricular volumes and mass using a MASS software package (MEDIS, Medical Imaging Systems, Leiden, the Netherlands). On end-diastolic images (first cine MR image after the R-wave trigger) and end-systolic images (cine MR image with visually the smallest cavity area), endocardial and epicardial contours of the right ventricle and left ventricle (LV) were obtained by manual tracing. Papillary muscles and trabeculae were included as part 
of the ventricular wall mass. Ventricular volumes were calculated using Simpson's rule. Stroke volume (SV) was calculated as end-diastolic volume (EDV) minus end-systolic volume (ESV). Right ventricular ejection fraction (RVEF) was calculated according to the following equation: (SV/EDV) $\times 100 \%$. For mass calculation, the myocardial volume was multiplied by the specific density of the heart $\left(1.05 \mathrm{~g} \cdot \mathrm{cm}^{-3}\right)[17]$. The relative ventricular wall thickness was calculated as the ratio of RV mass divided by the EDV [18]. Volume and mass measurements were indexed to BSA. RV end-systolic wall stress was calculated according to LaPlace's law (RV end-systolic wall stress $=0.5 \times \mathrm{RV}$ systolic pressure $\times \mathrm{RV}$ end-systolic radius/ RV end-systolic wall thickness), as explained previously [7, 19].

Six-minute walk test

The six-minute walking test (6MWT) was performed according to the American Thoracic Society guidelines [20].

\section{Blood sampling}

Since November 2002, N-terminal pro-brain natriuretic peptide (NT-proBNP) measurements have become part of our routine clinical assessment. NT-proBNP plasma levels were analysed using the Elecsys 1010 electrochemiluminescence immunoassay (Roche Diagnostics, Almere, the Netherlands), as described previously [21].

\section{Statistical analysis}

Statistical analyses were carried out using SPSS version 22 software (SPSS Inc., Chicago, IL, USA) or Prism 5 for Windows (GraphPad Software Inc., San Diego, CA, USA). A p-value $<0.05$ was considered statistically significant. Data are presented as mean \pm standard deviation for continuous variables and absolute for categorical variables, unless stated otherwise. Variables were log-transformed in cases of a non-normal distribution. Differences in baseline variables between patients treated with mono- or combination therapy were calculated using independent t-tests. Within-group differences in baseline and follow-up parameters were tested with paired t-tests. The changes in clinical parameters, haemodynamics and RV structure and function during follow-up were compared between the monotherapy and combination therapy group using linear regression analysis. This analysis was repeated with co-variate correction for differences in baseline values between groups (PVR). Correction for multiple testing was not applied because of our predefined study hypothesis and selected number of outcome parameters.

\section{Results}

\section{Patient characteristics}

Mean age of the total study population was $49 \pm 17$ years; $75 \%$ were female and the majority of patients had IPAH (85\%). There were no differences between the monotherapy and combination therapy groups with regard to age, gender, type of diagnosis, or NYHA class (table 1). Patients who received upfront combination therapy had a higher PVR and lower CI at baseline than patients initiated on monotherapy. No differences were found between the two groups with respect to NT-proBNP, exercise capacity or CMR RV parameters (table 2).

\section{Follow-up measurements}

The median time between baseline and follow-up measurements was 12 months (interquartile range 12 14 months). Both treatment regimens were associated with improvements in exercise capacity, NYHA class, haemodynamics and CMR variables after 1 year of follow-up (table 2, figures 2-4). The change in six-minute walk distance was greater in the combination group than in the monotherapy group $(\mathrm{p}=0.041)$. Furthermore, NT-proBNP levels decreased more in patients treated with combination therapy than in those who received monotherapy $(\mathrm{p}=0.001)$ (figure 2 ).

Both treatment groups showed a significant decrease in PVR, but the magnitude of decrease was larger in the upfront combination therapy group (combination versus monotherapy: $\mathrm{p}$ for change $<0.001$ ). MPAP decreased after combination therapy, but remained unchanged after monotherapy. Both groups showed a similar change in CI ( $p=0.071)$ (figure 3). RVEF improved more in the combination therapy group than in the monotherapy group $(\mathrm{p}<0.001)$. The mean change in right ventricular end-diastolic volume (RVEDV) was $-5 \pm 16 \mathrm{~mL} \cdot \mathrm{m}^{-2}$ after combination therapy and $3 \pm 16 \mathrm{~mL} \cdot \mathrm{m}^{-2}$ after monotherapy ( $\mathrm{p}$ for difference between groups $=0.038$ ). Patients who had received combination therapy had a more significant decrease in right ventricular end-systolic volume (RVESV) than patients who had received monotherapy (mean change: $-13 \pm 17 \mathrm{~mL} \cdot \mathrm{m}^{-2}$ versus $-1 \pm 15 \mathrm{~mL} \cdot \mathrm{m}^{-2} ; \mathrm{p}=0.002$ ) (figure 4 ). $\mathrm{RV}$ mass remained unchanged after monotherapy but decreased after combination therapy. The relative wall thickness of the right ventricle was unaltered in both treatment groups (table 2). 


\begin{tabular}{|c|c|c|c|c|}
\hline Variable & Total cohort & Monotherapy & Combination therapy & p-value \\
\hline Subjects $n$ & 80 & 45 & 35 & \\
\hline Female & $60(75)$ & $34(76)$ & $26(74)$ & 0.896 \\
\hline Age years & $49 \pm 17$ & $49 \pm 17$ & $50 \pm 19$ & 0.803 \\
\hline Diagnosis & & & & 0.103 \\
\hline Idiopathic PAH & $68(85)$ & 40 (89) & $28(80)$ & \\
\hline Heritable PAH & $10(13)$ & $3(7)$ & $7(20)$ & \\
\hline Drugs/toxins PAH & 2 (3) & $2(4)$ & 0 & \\
\hline NYHA class & & & & 0.067 \\
\hline II & $24(30)$ & $16(36)$ & $6(17)$ & \\
\hline III & $56(70)$ & $29(64)$ & $29(83)$ & \\
\hline BSA $\mathrm{m}^{2}$ & $1.9 \pm 0.2$ & $1.9 \pm 0.2$ & $1.9 \pm 0.7$ & 0.939 \\
\hline Comorbidities & & & & 0.158 \\
\hline Diabetes mellitus & $3(4)$ & $1(2)$ & $2(6)$ & \\
\hline Systemic hypertension & $14(18)$ & $5(11)$ & $9(26)$ & \\
\hline Coronary artery disease & $3(4)$ & $1(2)$ & $2(6)$ & \\
\hline \multicolumn{5}{|l|}{ Medical therapy } \\
\hline ERA & & $34(76)$ & $35(100)$ & \\
\hline Ambrisentan & $20(17)$ & $6(13)$ & 14 & \\
\hline Bosentan & $42(37)$ & $22(5)$ & $20^{\#}$ & \\
\hline Macitentan & 4 (3) & $3(7)$ & 1 & \\
\hline Sitaxentan & $3(3)$ & $3(7)$ & 0 & \\
\hline PDE5I & & $11(24)$ & $35(100)$ & \\
\hline Sildenafil & $31(27)$ & $9(20)$ & 22 & \\
\hline Tadalafil & $15(13)$ & $2(4)$ & 13 & \\
\hline \multicolumn{5}{|l|}{ Renal function } \\
\hline Creatinine $\mu \mathrm{mol} \cdot \mathrm{L}^{-1}$ & $84 \pm 23$ & $86 \pm 27$ & $81 \pm 18$ & 0.340 \\
\hline \multicolumn{5}{|c|}{$\begin{array}{l}\text { Data are presented as as mean } \pm \text { SD or } \mathrm{n}(\%) \text {, unless otherwise stated. PAH: pulmonary arterial } \\
\text { hypertension; NYHA: New York Heart Association; BSA: body surface area; ERA: endothelin receptor } \\
\text { antagonist; PDE5I: phosphodiesterase-5-inhibitor. \#: One patient did not tolerate the full dosage of } \\
\text { bosentan and therefore received a lower dose of } 62.5 \mathrm{mg} \text { twice daily. All other patients were treated with } \\
\text { full dosage of ERA and/or PDE5I. }\end{array}$} \\
\hline
\end{tabular}

The relative change in PVR was significantly correlated to the absolute change in RVEF after combination therapy $(\mathrm{R}=-0.60 ; \mathrm{p}<0.001)$. In contrast, we did not find a significant correlation between the change in PVR and RVEF after monotherapy ( $p>0.05$ ). Strikingly, RVEF decreased by $>3 \%$ [9] in only two out of 35 patients after initiation of combination therapy, and one of these two patients did not show an improved PVR either. In eight out of 45 patients (18\%) in the monotherapy group, RVEF decreased by $>3 \%$ despite therapy (figure 5).

In the monotherapy group, we did not find different therapeutic effects between the patients treated with ERA monotherapy and the patients treated with PDE5I ( $p>0.05$ for all parameters).

However, even after correction for baseline differences in PVR, we found significant differences in haemodynamics, CMR parameters and NT-proBNP between the combination therapy group and monotherapy group, except for RVEDV and RV mass (table S1).

\section{Treatment strategies}

In the group of patients initiated on monotherapy, one patient switched from ERA (bosentan) to PDE5I (sildenafil; after 4 months) owing to liver enzyme abnormalities. All other patients in the upfront monotherapy group received and tolerated the full dosage of ERA or PDE5I. In the combination therapy group, one patient did not tolerate the full dosage of the combined treatment of bosentan plus sildenafil owing to liver enzyme abnormalities; therefore, the dosage of bosentan was kept constant at $62.5 \mathrm{mg}$ twice daily. All other patients in the combination therapy group were treated with full-dosage ERA plus PDE5I.

In the monotherapy group, eight patients $(18 \%)$ were treated with sequential combination therapy $(n=7$ initiated on ERA and $n=1$ initiated on PDE5I) owing to lack of clinical improvement after upfront monotherapy (median time 5 months; IQR 5-6 months). In the group of patients initiated on dual combination therapy, treatment was switched to sequential triple therapy (i.e. by adding prostacyclin) in 
TABLE 2 Differences in clinical parameters at baseline and during follow-up

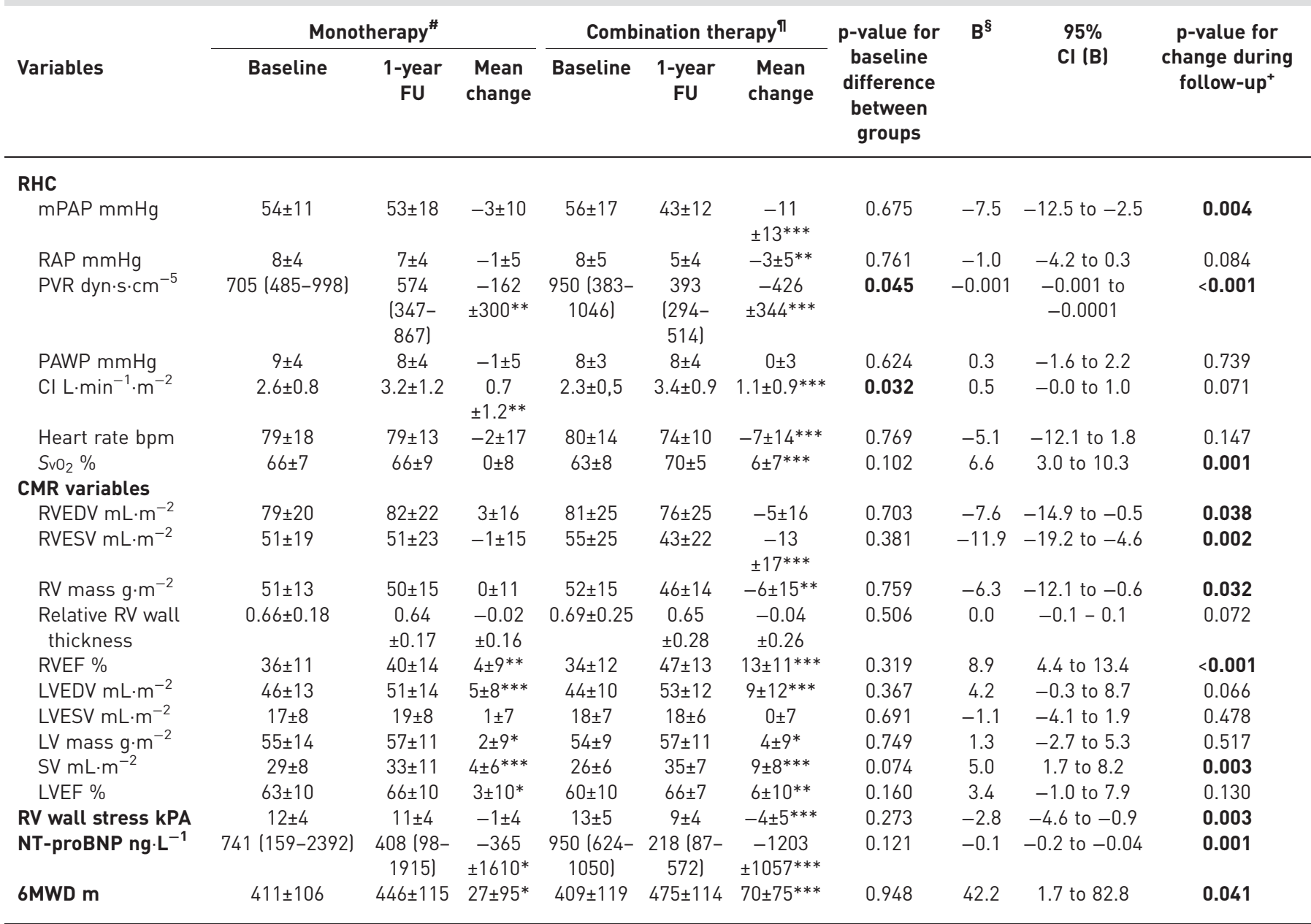

Data are presented as mean \pm SD or median (IQR), unless otherwise stated. ${ }^{\#}: n=45 ;{ }^{n}: n=35 ;{ }^{+}: p$-value for the difference in change in variables between groups during follow-up without correction for baseline co-variates; ${ }^{\S}$ : B represents the regression coefficient for the difference in variable change between groups without correction for baseline co-variates. Within-group differences between baseline and follow-up variables are indicated by ${ }^{*}: p<0.05 ;{ }^{* *}: p<0.01 ;{ }^{* * *}: p<0.001$. NT-proBNP was measured in 71 patients and values were log-transformed before testing. FU: follow-up; RHC: right-sided heart catheterisation; mPAP: mean pulmonary arterial pressure; RAP: right atrial pressure; PVR: pulmonary vascular resistance; PAWP: pulmonary arterial wedge pressure; $\mathrm{Cl}$ : cardiac index; $\mathrm{SvO}_{2}$ : mixed venous oxygen saturation; CMR: cardiac magnetic resonance imaging; RVEDV: right ventricular end diastolic volume; RVESV: right ventricular end-systolic volume; RV: right ventricle; RVEF: right ventricular ejection fraction; LVEDV: left ventricular end-diastolic volume; LVESV: left ventricular end-systolic volume; LV: left ventricle; SV: stroke volume; LVEF: left ventricular ejection fraction; NT-proBNP: N-terminal pro-brain natriuretic peptide; 6MWD: six-minute walk distance. Values presented in bold are statistically significant.

three patients after 3,8 and 10 months, respectively, owing to clinical worsening (i.e. deterioration into NYHA class IV). In table S2, an analysis is provided of the data after exclusion of the eight patients from the monotherapy group and the three patients from the combination therapy who had switched therapy during the 12 months of follow-up. The remaining 37 patients of the upfront monotherapy group showed differences in haemodynamics, NT-proBNP and CMR parameters compared with the 32 patients in the upfront combination therapy group. The results from table S2 are comparable to those shown in table 1 and table S1.

\section{Discussion}

In the present study, we showed - in NYHA class II and III PAH patients - that treatment with upfront combination therapy ERA plus PDE5I is associated with significant reductions in PVR and pulmonary pressures and resulted in improved RV volumes and function. Compared with combination therapy, upfront monotherapy was associated with smaller decreases in RV afterload, and RV volumes remained unchanged. 

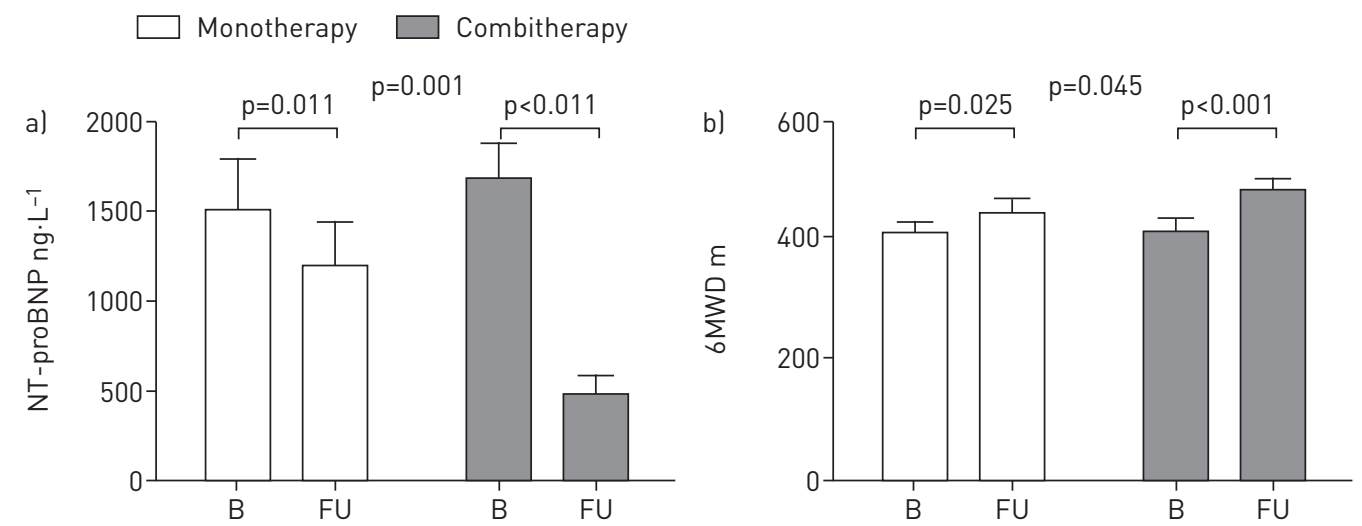

FIGURE 2 a) The decrease in N-terminal pro-brain natriuretic peptide (NT-proBNP) was greater in the upfront combination therapy group (grey bars) than in the monotherapy group (white bars). b) The six-minute walk distance (6MWD) improved in both groups. Data are presented as mean \pm SEM. B: baseline; FU: follow-up.

\section{Effects of combination therapy on RV afterload}

In accordance with earlier studies [22], we found statistically significant reductions in PVR after single-agent therapy, but the PVR change was modest $(\sim 19 \%)$ and mPAP was not significantly reduced. In contrast, we showed that PVR dropped by $\sim 59 \%$ after upfront oral combination therapy. These results are in agreement with former studies showing a $\sim 45-70 \%$ decrease in PVR after upfront therapy of ERA plus PDE5I [8] or ERA plus prostacyclins $[23,24]$. Importantly, the decrease in PVR after combination therapy was not only accompanied by normalisation of the $\mathrm{CO}$ but also by a $\sim 23 \%$ reduction in mPAP. This RV unloading was accompanied by subsequent improvements in RV remodelling and function. In fact, a progressive deterioration in RVEF was found in only two patients treated with upfront combination therapy.
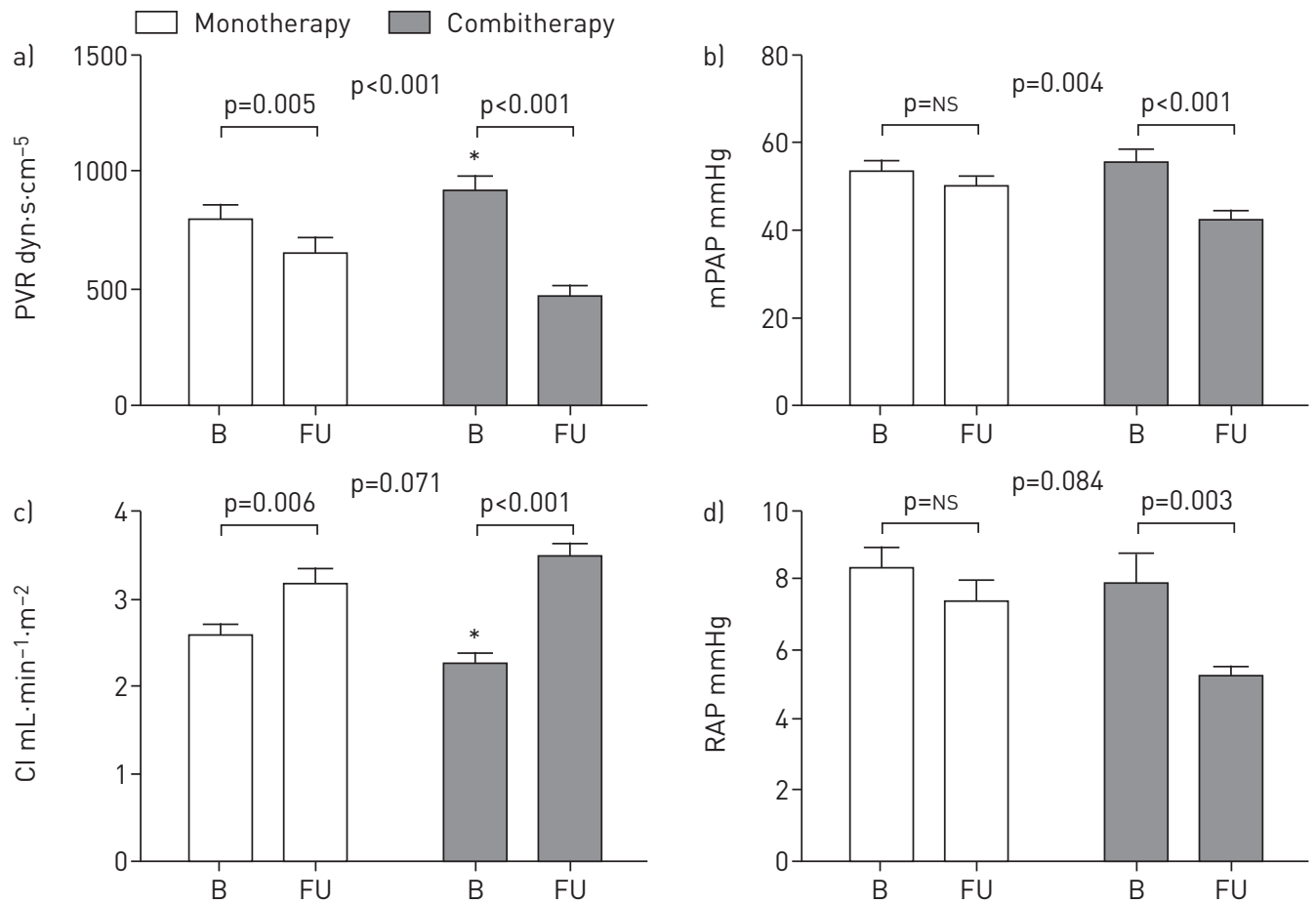

FIGURE 3 Patients treated with upfront combination therapy (grey bars) showed greater improvements in (a) pulmonary vascular resistance (PVR) and (b) mean pulmonary arterial pressure (mPAP) than patients receiving upfront monotherapy (white bars). Both patients groups showed normalisation of the cardiac index (CI) (c). Right atrial pressure (RAP) (d) remained unchanged after monotherapy and improved after combination therapy. Data are presented as mean \pm SEM. ${ }^{*}: p<0.05$ for baseline difference between the mono and combination therapy groups. B: baseline; FU: follow-up; Ns: nonsignificant. 

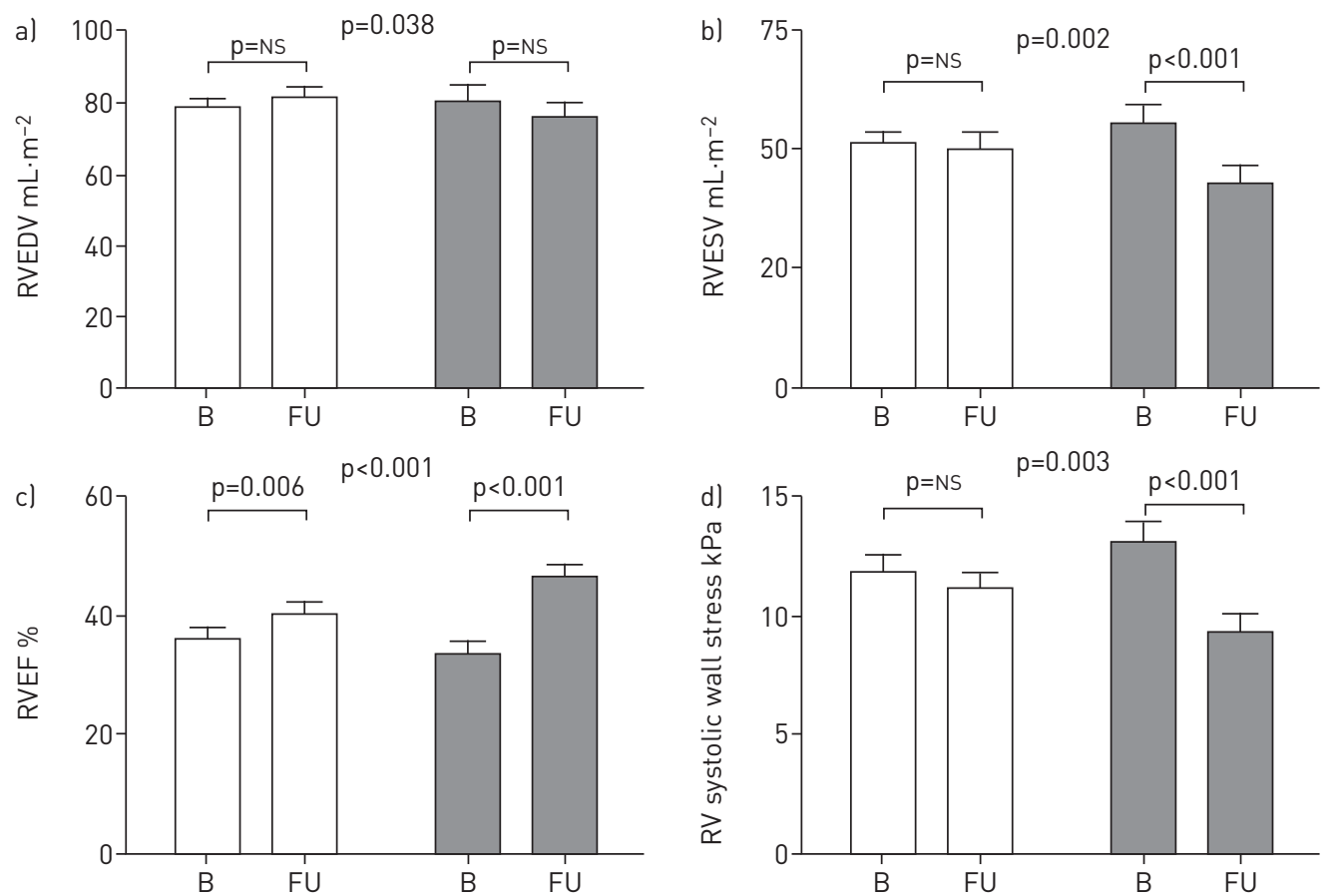

FIGURE 4 Neither monotherapy (white bars) nor combination therapy (grey bars) resulted in an improvement in right ventricular end-diastolic volume (RVEDV) (a). Right ventricular end-systolic volume (RVESV) (b) and right ventricular (RV) wall stress (d) decreased after combination therapy but remained unaltered after monotherapy. The increase in right ventricular ejection fraction (RVEF) (c) was greater after combination therapy than after monotherapy. Data are presented as mean \pm SEM. B: baseline; FU: follow-up; NS: nonsignificant.

\section{Effects of upfront combination therapy on RV volumes}

The most important finding of the present study was that RVESV decreased significantly after upfront combination therapy. Of note, a decrease in RVESV was accompanied by a stable RVEDV, and consequently stroke volume was improved after combination treatment.

In line with previous studies, we showed that RV volumes remain unchanged after upfront monotherapy [11-15]. Moreover, it has been shown that RV volumes do not alter after combination therapy when

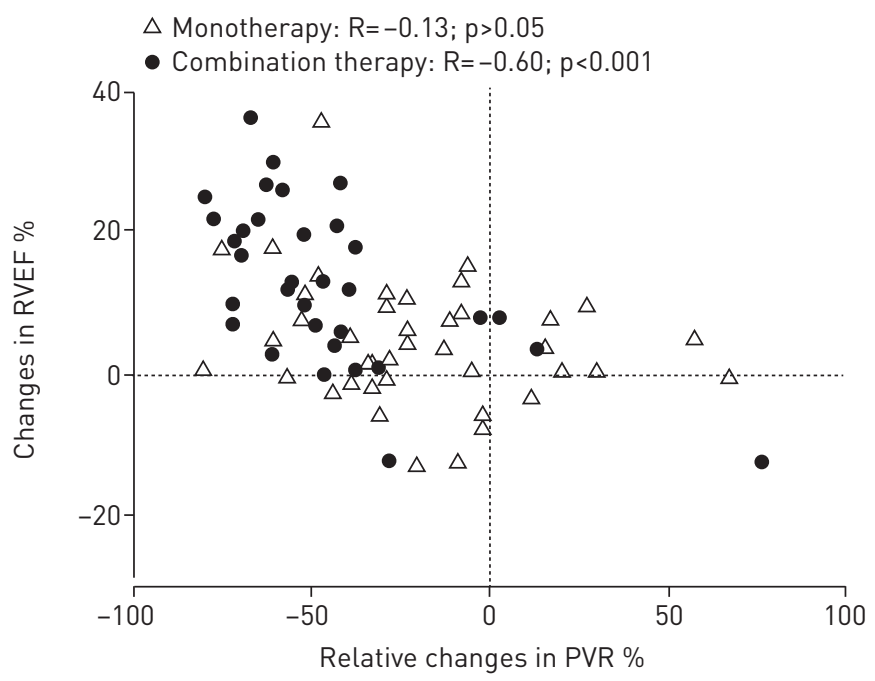

FIGURE 5 The relative changes in pulmonary vascular resistance (PVR) were correlated with the changes in right ventricular ejection fraction (RVEF) after upfront combination therapy (black circles) but not after upfront monotherapy lopen triangles). 
applied sequentially during follow-up [12]. Our findings are of major clinical relevance since RV dilatation is one of the most important prognostic predictors in patients with $\mathrm{PAH}[25,26]$ and is a sensitive parameter for monitoring patients during follow-up [18]. In addition, and in line with earlier studies [11], we found that changes in RV mass are relatively small after medical treatment. Small changes in RV mass with concomitant large reductions in RV volumes after combination therapy contribute to a more favourable concentric RV remodelling pattern which is associated with better survival [27, 28].

\section{Possible mechanisms for improved RV adaptation and function after upfront combination therapy}

In this study, we evaluated the relationship between NT-proBNP, RV afterload and RV remodelling in $\mathrm{PAH}$ patients receiving combination therapy. In accordance with the results from the AMBITION trial [5] and other studies $[8,29]$, we observed a $\sim 77 \%$ reduction in NT-proBNP after upfront combination therapy. The magnitude of the decrease in NT-proBNP was significantly larger after combination therapy than after monotherapy. These findings are not only of prognostic relevance [30, 31] but also of physiological interest since NT-proBNP is considered a surrogate marker of RV wall stress [21, 32]. According to LaPlace's law, ventricular wall stress can be reduced by either reducing intraluminal pressures, decreasing chamber radius, or increasing wall thickness [19]. In the present study, we showed that combination therapy resulted in significant reductions in both PVR and RV pressures, leading to a considerable decrease in intraluminal RV volumes and smaller changes in RV wall thickness. As a consequence, the calculated RV end-systolic wall stress dropped significantly. In contrast, after monotherapy pulmonary pressures, RV volumes and mass were unchanged, and thereafter in the monotherapy group, RV wall stress remained high. We showed that the changes in RV pressures and volumes after medical treatment were the most important contributors to changes in RV wall stress, and are summarised in figure 6. Our findings are of clinical importance since a decreased RV wall stress positively affects myocardial perfusion and oxygen demand $[36,37]$ and may therefore contribute to the reversal of progressive RV dysfunction. This is supported by the large and clinically relevant increase in RVEF observed after combination therapy [9]. Moreover, we observed a significant relationship between

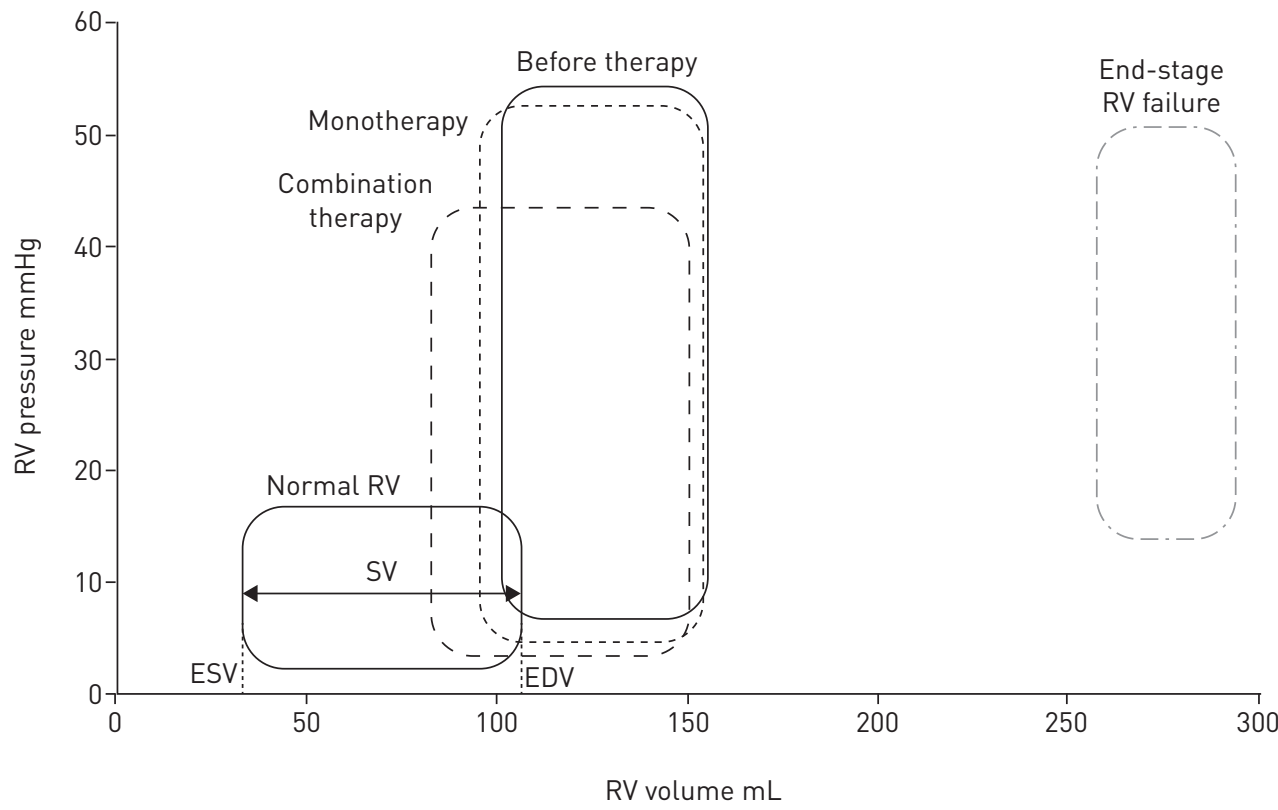

FIGURE 6 Schematic pressure-volume relationships that summarise the effects of upfront monotherapy (----) and combination therapy (- - ) in patients with pulmonary arterial hypertension (PAH) (-). Compared with control subjects (Normal RV) [33,34], PAH patients showed increased right ventricular (RV) pressures and right ventricular end-systolic volume (RVESV). After monotherapy, RV pressures were modestly reduced but right ventricular end-diastolic volume (RVEDV) and RVESV remained unchanged. Importantly, upfront combination therapy was associated with significant reductions in both RV pressures and RVESV. Because RV pressures and volumes are main contributors to RV wall stress, the calculated ventricular systolic wall stress will be lowered after combination therapy but remains high after monotherapy. The pressure-volume loop of end-stage RV failure (.-.) is included for comparison. In cases of RV failure, RV pressures are comparable to other stages of RV dysfunction, though RV volumes are massively increased [18, 35]. Of note, stroke work (i.e. area within one pressure-volume loopl is increased in $\mathrm{PAH}$ patients compared with controls but depresses with the development of RV failure. EDV: end-diastolic volume; ESV: end-systolic volume; SV: stroke volume. 
the changes in PVR and RVEF after combination therapy, but did not find such a relationship after monotherapy. These results demonstrate that in cases where there is a sufficient decrease in RV afterload, reversal of RV dysfunction is guaranteed and "right ventricle - arterial coupling" will be preserved [38], whereas smaller changes in PVR do not necessarily lead to improved RV function. In addition to a reduced afterload and potential direct RV therapeutic effects, other intrinsic RV factors could also play a role in a more favourable RV adaptation response following combination therapy [39].

\section{Clinical implications}

We have shown - in patients with PAH - that upfront oral combination therapy, in contrast to upfront monotherapy, is associated with a significant reduction in the main determinants of wall stress - namely, RV volumes and mPAP. Since progressive RV dilation and increased RV wall stress precede ultimate disease progression and are major contributing factors that drive the right ventricle into failure $[6,18]$, the results of this retrospective analysis suggest that the vicious circle leading to the development of RV failure might be interrupted by aggressive upfront medical treatment in all patients. In addition, the favourable RV therapeutic effects observed in this study also raise the question as to whether prognosis can be improved by a goal-oriented strategy specifically directed at improving RV remodelling and function.

\section{Limitations}

The present study has the limitations of a retrospective analysis. The treatment strategy was non-controlled and rather reflects the therapeutic choice of the treating physician. However, our study reflected the treatment strategy that is generally accepted in clinical care and is supported by official guidelines [3]. Furthermore, the therapeutic effects on haemodynamics and NT-proBNP observed after both upfront monotherapy and combination therapy correspond to earlier prospective studies indicating that our results reflect "real world" therapeutic responses $[5,29]$ In addition, our study may be biased by the lengthy inclusion period - from 2002 to 2015 - while only monotherapy was available before 2005 . Yet, we would like to emphasise that the present study was not aimed at measuring treatment efficacy. Patients receiving upfront combination therapy were slightly more compromised at baseline than patients treated with monotherapy. However, we limited this potential bias by correcting for differences in baseline characteristics.

\section{Conclusions}

We showed - in NYHA class II and III PAH patients - that treatment with upfront combination therapy of an ERA plus a PDE5I is associated with significant reductions in RV volumes. This was accompanied by reduced NT-proBNP levels and a relief of the calculated RV wall stress.

\section{Acknowledgements}

All authors have made substantial contributions to conception and design, acquisition, analysis or interpretation of the data, and read and revised the manuscript critically. All authors gave their final approval.

\section{References}

1 Voelkel NF, Quaife RA, Leinwand LA, et al. Right ventricular function and failure: report of a National Heart, Lung, and Blood Institute working group on cellular and molecular mechanisms of right heart failure. Circulation 2006; 114: 1883-1891.

2 McLaughlin VV, McGoon MD. Pulmonary arterial hypertension. Circulation 2006; 114: 1417-1431.

3 Galie N, Humbert M, Vachiery JL, et al. 2015 ESC/ERS Guidelines for the diagnosis and treatment of pulmonary hypertension. Eur Respir J 2015; 46: 903-975.

4 McLaughlin VV, Gaine SP, Howard LS, et al. Treatment goals of pulmonary hypertension. J Am Coll Cardiol 2013; 62: D73-D81.

5 Galie N, Barbera JA, Frost AE, et al. Initial use of ambrisentan plus tadalafil in pulmonary arterial hypertension. N Engl J Med 2015; 373: 834-844.

6 Grossman W, Jones D, McLaurin LP. Wall stress and patterns of hypertrophy in the human left ventricle. J Clin Invest 1975; 56: 56-64.

7 Mauritz GJ, Vonk-Noordegraaf A, Kind T, et al. Pulmonary endarterectomy normalizes interventricular dyssynchrony and right ventricular systolic wall stress. J Cardiovasc Magn Reson 2012; 14: 1-9.

8 Sitbon O, Sattler C, Bertoletti L, et al. Initial dual oral combination therapy in pulmonary arterial hypertension. Eur Respir J 2016; 47: 1727-1736.

9 van de Veerdonk MC, Kind T, Marcus JT, et al. Progressive right ventricular dysfunction in patients with pulmonary arterial hypertension responding to therapy. J Am Coll Cardiol 2011; 58: 2511-2519.

10 Vonk Noordegraaf A, Westerhof N. Right ventricular ejection fraction and NT-proBNP are both indicators of wall stress in pulmonary hypertension. Eur Respir J 2007; 29: 622-623.

11 Peacock AJ, Crawley S, McLure L, et al. Changes in right ventricular function measured by cardiac magnetic resonance imaging in patients receiving pulmonary arterial hypertension-targeted therapy: the EURO-MR study. Circ Cardiovasc Imaging 2014; 7: 107-114.

12 van Wolferen SA, Boonstra A, Marcus JT, et al. Right ventricular reverse remodelling after sildenafil in pulmonary arterial hypertension. Heart 2006; 92: 1860-1861. 
13 Wilkins MR, Paul GA, Strange JW, et al. Sildenafil versus Endothelin Receptor Antagonist for Pulmonary Hypertension (SERAPH) study. Am J Respir Crit Care Med 2005; 171: 1292-1297.

14 Galie N, Hinderliter AL, Torbicki A, et al. Effects of the oral endothelin-receptor antagonist bosentan on echocardiographic and doppler measures in patients with pulmonary arterial hypertension. J Am Coll Cardiol 2003; 41: 1380-1386

15 Hinderliter AL, Willis PW 4th, Barst RJ, et al. Effects of long-term infusion of prostacyclin (epoprostenol) on echocardiographic measures of right ventricular structure and function in primary pulmonary hypertension. Circulation 1997; 95: 1479-1486.

16 Roeleveld RJ, Vonk-Noordegraaf A, Marcus JT, et al. Effects of epoprostenol on right ventricular hypertrophy and dilatation in pulmonary hypertension. Chest 2004; 125: 572-579.

17 Katz J, Whang J, Boxt LM, et al. Estimation of right ventricular mass in normal subjects and in patients with primary pulmonary hypertension by nuclear magnetic resonance imaging. J Am Coll Cardiol 1993; 21: 1475-1481.

18 van de Veerdonk MC, Marcus JT, Westerhof N, et al. Signs of right ventricular deterioration in clinically stable patients with pulmonary arterial hypertension. Chest 2015; 147: 1063-1071.

19 Westerhof N, Stergiopulos N, Noble MIM. Snapshots of hemodynamics: an aid for clinical research and graduate education. 2nd Edn. New York, Springer, 2010.

20 American Thoracic Society. ATS statement: guidelines for the six-minute walk test. Am J Respir Crit Care Med 2002; 166: 111-117.

21 Gan CT, McCann GP, Marcus JT, et al. NT-proBNP reflects right ventricular structure and function in pulmonary hypertension. Eur Respir J 2006; 28: 1190-1194.

22 Galie N, Manes A, Negro L, et al. A meta-analysis of randomized controlled trials in pulmonary arteria hypertension. Eur Heart J 2009; 30: 394-403.

23 Kemp K, Savale L, O'Callaghan DS, et al. Usefulness of first-line combination therapy with epoprostenol and bosentan in pulmonary arterial hypertension: an observational study. J Heart Lung Transplant 2012; 31: 150-158.

24 Humbert M, Barst RJ, Robbins IM, et al. Combination of bosentan with epoprostenol in pulmonary arterial hypertension: BREATHE-2. Eur Respir J 2004; 24: 353-359.

25 Swift AJ, Rajaram S, Campbell MJ, et al. Prognostic value of cardiovascular magnetic resonance imaging measurements corrected for age and sex in idiopathic pulmonary arterial hypertension. Circ Cardiovasc Imaging 2014; 7: 100-106.

26 van Wolferen SA, Marcus JT, Boonstra A, et al. Prognostic value of right ventricular mass, volume, and function in idiopathic pulmonary arterial hypertension. Eur Heart J 2007; 28: 1250-1257.

27 Badagliacca R, Poscia R, Pezzuto B, et al. Right ventricular concentric hypertrophy and clinical worsening in idiopathic pulmonary arterial hypertension. J Heart Lung Transplant 2016; 35: 1321-1329.

28 Vonk-Noordegraaf A, Haddad F, Chin KM, et al. Right heart adaptation to pulmonary arterial hypertension: physiology and pathobiology. J Am Coll Cardiol 2013; 62: D22-D33.

29 Hassoun PM, Zamanian RT, Damico R, et al. Ambrisentan and tadalafil up-front combination therapy in scleroderma-associated pulmonary arterial hypertension. Am J Respir Crit Care Med 2015; 192: 1102-1110.

30 Leuchte $\mathrm{HH}$, El Nounou M, Tuerpe JC, et al. N-terminal pro-brain natriuretic peptide and renal insufficiency as predictors of mortality in pulmonary hypertension. Chest 2007; 131: 402-409.

31 Mauritz GJ, Rizopoulos D, Groepenhoff H, et al. Usefulness of serial N-terminal pro-B-type natriuretic peptide measurements for determining prognosis in patients with pulmonary arterial hypertension. Am J Cardiol 2011; 108: $1645-1650$.

32 Blyth KG, Groenning BA, Mark PB, et al. NT-proBNP can be used to detect right ventricular systolic dysfunction in pulmonary hypertension. Eur Respir J 2007; 29: 737-744.

33 van de Veerdonk MC, Dusoswa SA, Marcus JT, et al. The importance of trabecular hypertrophy in right ventricular adaptation to chronic pressure overload. Int J Cardiovasc Imaging 2014; 30: 357-365.

34 Kawut SM, Barr RG, Lima JA, et al. Right ventricular structure is associated with the risk of heart failure and cardiovascular death: the Multi-Ethnic Study of Atherosclerosis (MESA)-right ventricle study. Circulation 2012; 126: $1681-1688$.

35 Ryo K, Goda A, Onishi T, et al. Characterization of right ventricular remodeling in pulmonary hypertension associated with patient outcomes by 3-dimensional wall motion tracking echocardiography. Circ Cardiovasc Imaging 2015; 8: 1-9.

36 Westerhof N, Boer C, Lamberts RR, et al. Cross-talk between cardiac muscle and coronary vasculature. Physiol Rev 2006; 86: 1263-1308.

37 Gomez A, Bialostozky D, Zajarias A, et al. Right ventricular ischemia in patients with primary pulmonary hypertension. J Am Coll Cardiol 2001; 38: 1137-1142.

38 Vonk-Noordegraaf A, Westerhof N. Describing right ventricular function. Eur Respir J 2013; 41: $1419-1423$.

39 Voelkel NF, Gomez-Arroyo J, Abbate A, et al. Mechanisms of right heart failure-a work in progress and a plea for failure prevention. Pulm Circ 2013; 3: 137-143 